\title{
A Superconducting Focusing Solenoid for the Neutrino Factory Linear Accelerator
}

\author{
M. A. Green, V. Lebedev, and B. P. Strauss
}

\begin{abstract}
The proposed superconducting linear accelerator at accelerates muons from $190 \mathrm{MeV}$ to $2.45 \mathrm{GeV}$ will use superconducting solenoids for focusing the muon beam. The accelerator will use superconducting RF cavities. These cavities are very sensitive to stay magnetic field from the focusing magnets. Superconducting solenoids can have large stray fields. This paper describes the 201.25-MHz acceleration system for the neutrino factory. This paper also describes a focusing solenoid that delivers almost no stray field to a neighboring superconducting RF cavity.
\end{abstract}

Index Terms-Active shielding, superconducting focusing solenoid, superconducting RF cavities.

\section{INTRODUCTION}

$\mathbf{T}$ HE PROPOSED neutrino factory generates neutrinos from muon decay in a muon storage ring [1], [2]. The muons are created from the decay of pions produced from the collision of protons on a fixed target. The pions are captured by a 20-T solenoid. The pions then decay to muons as they pass down a long channel. The muons are phase-rotated, bunched, and cooled before they can be accelerated and stored in the storage ring [3].

The first stage of acceleration is a linear accelerator that uses superconducting RF cavities [4]. Muon focusing during this acceleration occurs within superconducting solenoids. After the muons are accelerated to $2350 \mathrm{MeV}$, they enter a recirculating linac that accelerates them to their final energy of $20 \mathrm{GeV}$. The $20-\mathrm{GeV}$ muons are then stored in storage ring where they decay to two neutrinos and an electron or positron (depending on the charge of the originating muon type).

\section{FOCUSING SOLENOIDS FOR THE LINEAR ACCELERATOR}

The proposed linear accelerator that accelerates the muons from $190 \mathrm{MeV}$ to $2350 \mathrm{MeV}$ extends nearly $400 \mathrm{~m}$. The linear accelerator will use high gradient superconducting RF cavities to accelerate the beam. These cavities will not only increase the acceleration gradient, but they will also save electric power. Between the RF cavities there will be superconducting solenoids that provide beam focusing.

Manuscript received September 26, 2001. This work was supported by the Office of Science, United States Department of Energy under DOE Contract DE-AC03-76SF00098.

M. A. Green is with the Lawrence Berkeley National Laboratory, Berkeley, CA 94720 USA.

V. Lebedev is with the Thomas Jefferson National Laboratory, Newport News, VA 23606 USA.

B. P. Strauss is with the U.S. Department of Energy, Germantown, MD 20874 USA.

Publisher Item Identifier S 1051-8223(02)03475-9.
The 400-m long linear accelerator is divided into three sections. Upstream from the linear accelerator is a matching section that provides the first $60 \mathrm{MeV}$ of muon acceleration. The end of the matching section consists of a pair of low stray field solenoids with adjustable currents for tuning. The first section has four short modules that are 5-m long. Each short module has two cells of RF cavities. The second section has 16 medium length modules that are 8-m long. Each intermediate module has two cells of RF cavities arranged in groups of two with a $1-\mathrm{m}$ space between groups. The short and intermediate modules have a $1-\mathrm{m}$ long focusing solenoid with a $1.25-\mathrm{m}$ long straight section at each end. The third acceleration section has 19 long modules that are 13-m long. The long module has eight superconducting RF cells arranged in groups of two with a 1-m space between cell groups. The focusing solenoid is $1.5 \mathrm{~m}$ long with a 1.25-m long space separating it from the adjacent RF cavity cells. The three types of acceleration modules used for the linear accelerator are illustrated in Fig. 1.

Unlike focusing quadrupoles, focusing solenoids can produce a stray field that exists some distance from the magnet. The superconducting RF cavities are sensitive to magnetic fields even at the level of $0.0001 \mathrm{~T}$ (versus $0.043 \mathrm{~T}$ for the smallest single solenoid in Table I), so a key parameter in the focusing solenoid design is getting rid of the solenoid stray field in the RF cavities. The methods one can use to eliminate the stray field in the RF cavities are: 1) The focusing solenoid should produce zero net magnetic moment [5], [6]. This means that the coil that produces the field is bucked by a coil that is larger in diameter. 2) The field from the bucking coils should be distributed in the same way as the solenoid field. This suggests that the bucking solenoid be around the focusing solenoid so that the return flux from the focusing solenoid is returned between the focusing solenoid and the bucking solenoid [7]. 3) The nested solenoids should be surrounded by iron except where the muon beam passes through the solenoid. 4) An iron flux shield should be installed between the focusing solenoid and the RF cavities. 5) If the stray field is still not low enough, the RF cavities can be covered with a type 2 superconducting shield. This shield will trap Earth's magnetic field, but it will shield out the remaining stray flux from a nearby solenoid provided the cavities are cold (below $5 \mathrm{~K}$ ) when the solenoid is turned on. An iron shielded actively shielded solenoid appears to be a minimum requirement in order to reduce the stray field to an acceptable level.

Fig. 1 shows the location of iron flux barriers that provide additional magnetic shielding for the nearby RF cavities. The barrier is designed to remove most of the magnetic flux that passes through the beam hole in the iron shield around the superconducting focusing solenoid. 


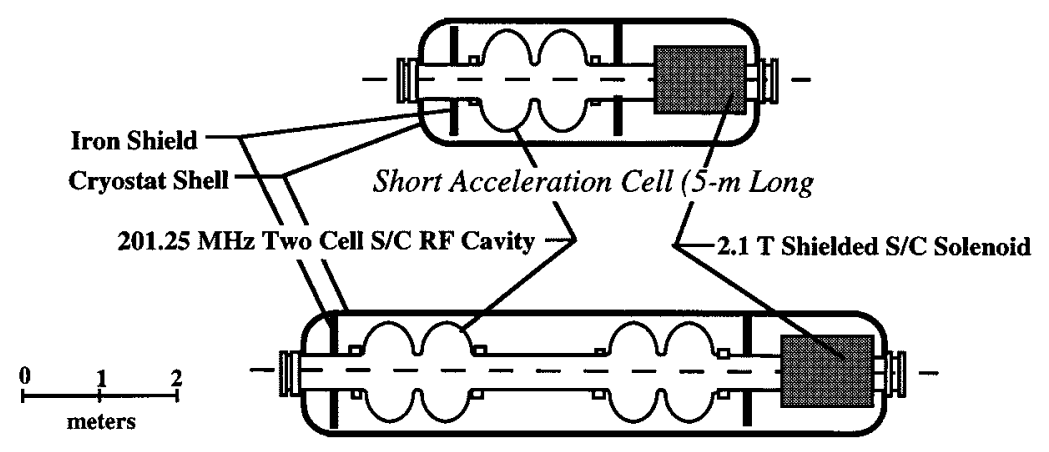

Intermediate Acceleration Cell (8-m Long)

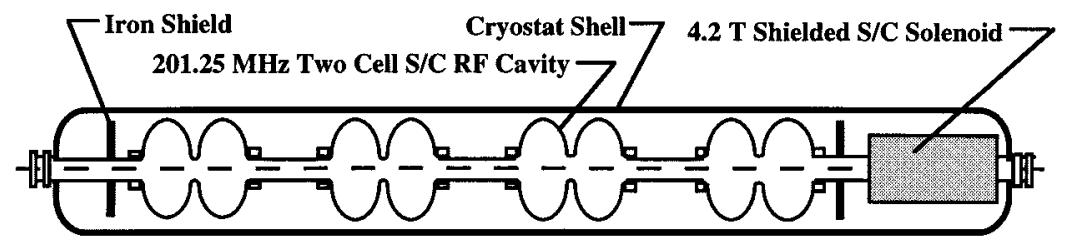

Long Acceleration Cell (13-m Long)

Fig. 1. The three linear acceleration cells for the superconducting linac. Shown in the figure are the superconducting RF cavities and the supperconducting solenoid magnets used for focusing. No detail is shown concerning the solenoids.

TABLE I

PARAMETERS FOR THE FOCUSING SOLENOIDS

\author{
Beam Bore Diameter (mm) \\ Iron Shell Length (mm) \\ Iron Shell Outer Diameter (mm) \\ Iron Shell Thickness (mm) \\ Inner Coil Average Radius (mm) \\ Inner Coil Thickness (mm) \\ Number of Inner Coil Layers/Turns \\ Outer Coil Average Radius (mm) \\ Outer Coil Thickness (mm) \\ Outer Coil Center Gap (mm) \\ Number of Outer Coil Layers/Turns \\ Solenoid Cold Mass (kg) \\ Solenoid Cryostat Mass $(\mathrm{kg})$ \\ Iron Shell Mass (kg) \\ Solenoid Average Magnetic Induction (T) \\ Solenoid Magnetic Length $(\mathrm{m})$ \\ Magnet Design Current (A) \\ Peak Induction in the Inner Coil $\mathrm{Bp}(\mathrm{T})$ \\ Magnet Conductor Ic at $4.4 \mathrm{~K}$ and $\mathrm{Bp}$ (A) \\ S/C Current Density (MA m ${ }^{-2}$ ) \\ Solenoid Stored Energy (MJ) \\ Solenoid Self Inductance (H) \\ Quench Protection $E \mathrm{~J}^{2}$ Limit $\left(\mathrm{A}^{2} \mathrm{~m}^{-4} \mathrm{~J}\right)$
}

$\begin{array}{ccc}\text { Short Cell } & \text { Medium Cell } & \text { Long Cell } \\ 460 & 460 & 300 \\ 1300 & 1300 & 1750 \\ 1240 & 1240 & 1120 \\ 9.5 & 9.5 & 9.5 \\ 254 & 254 & 182 \\ 10.4 & 10.4 & 31.2 \\ 8 / 4840 & 8 / 4840 & 24 / 21816 \\ 520.6 & 520.6 & 453.6 \\ 2.6 & 2.6 & 5.2 \\ 50 & 50 & 50 \\ 2 / 1152 & 2 / 1152 & 4 / 3512 \\ 376 & 376 & 746 \\ 166 & 166 & 238 \\ 485 & 485 & 581 \\ 2.1 & 2.1 & 4.2 \\ \sim 1.0 & \sim 1.0 & \sim 1.5 \\ 469.6 & 469.6 & 274.0 \\ \sim 2.9 & \sim 2.9 & \sim 5.8 \\ \sim 1100 & \sim 1100 & \sim 590 \\ 307 & 307 & 180 \\ 0.421 & 0.421 & 1.306 \\ 3.82 & 3.82 & 34.8 \\ 3.97 \times 10^{22} & 3.97 \times 10^{22} & 4.23 \times 10^{22}\end{array}$

Table I presents the mechanical and electrical parameters for the short, medium, and long module focusing solenoids. The matching solenoids at the start of the channel are similar to the first focusing solenoids. All of these solenoids are designed to have zero net magnetic moment. The solenoids in Table I are assumed to have a warm bore and a warm iron shell around the solenoid pair. It should be noted that the magnet bore does not have to be warm. The solenoid pair is assumed to be powered through a single set of HTS and gas-cooled electrical leads.
Since the magnet pair is hooked in series, the focusing solenoids will have nearly zero net magnetic moment at all excitation currents.

The design of the solenoid and its bucking coil is a case where the cost of the magnet is to be minimized. A bucking coil that has too small a diameter will create a large bucking field. More superconductor is needed in the inner solenoid in order to produce the desired on axis field. A bucking coil that is too large will result in a magnet system with more overall mass even though that 
magnet may have a smaller amount of superconductor within it. A case that is close to optimum is a case where the bucking coil has twice the radius of the inner coil. The magnets that are shown in Table I were designed to have an even number of layers of superconductor in both coils. There is a gap in the outer coil that allows one to attach current leads.

Fig. 2 shows a half cross section of the long focusing solenoid (1.5-m long with $4.2 \mathrm{~T}$ in the inner bore) in a plane that goes along the solenoid axis. Fig. 2 shows the separation of the inner coil and the bucking coil. Also shown are the magnet cryostat, an electrical lead, and the iron shield around the actively shielded solenoid. The center of the cryostat has no iron shield around it because there is very little magnetic flux leaking outside the bucking solenoid. The magnet shown in Fig. 2 is two concentric solenoids. A superconducting pancake at the end of the magnet may be more effective in shielding the nearby RF cavity. In any event the net magnetic moment should be zero.

The solenoid shown in Fig. 2 has the inner solenoid mounted on the inside of an aluminum shell. The outer coil is wound on the outside of an aluminum bobbin.

The conductor proposed for the focusing solenoid insulated dimensions of 1.0 by $1.65 \mathrm{~mm}$. This is a standard MRI conductor with a copper to superconductor ratio of 4 . The conductor contains $5580-\mu \mathrm{m}$ filaments of niobium-titanium. The conductor twist pitch is $12.7 \mathrm{~mm}$. The proposed conductor has a guaranteed critical current of $760 \mathrm{~A}$ at $4.2 \mathrm{~K}$ and $5 \mathrm{~T}$. The turn to turn insulation on the proposed conductor is a varnish. The layer to layer insulation proposed for the magnet is about $0.35 \mathrm{~mm}$ of epoxy impregnated fiberglass. The magnet coil can either be wound and then vacuum impregnated or it could be wound as a wet lay-up with a room temperature cure.

\section{COOLING OF THE Focusing SOLENOID}

The focusing solenoids are cooled by conduction from the 6061-aluminum support structure. The aluminum support structure will be cooled by two-phase helium flowing in tubes attached to it. It is assumed that all of the magnets in an acceleration section are cooled in series from the two-phase helium refrigerator and control cryostat. Whether this refrigerator is the same one that cools the superconducting RF cavities depends on the operating temperature of the RF cavities. The $4.4 \mathrm{~K}$ heat load is about $0.5 \mathrm{~W}$ for the short solenoid. The long solenoid heat leak is about $0.7 \mathrm{~W}$. One can cool 20 magnets with a two-phase helium a mass flow rate of $2.5 \mathrm{~g} \mathrm{~s}^{-1}$. The two-phase helium cooling tubes will be attached to the coil support structures, the attachment points for the cold mass supports, and the base of the HTS leads.

The heat load into the shield circuit helium stream is expected to vary from 6.1 to $7.3 \mathrm{~W}$ depending on the length of the magnet. The shield gas comes from the refrigerator at a temperature of $30 \mathrm{~K}$. This gas enters the magnet cryostat through a single vacuum insulated tube. The helium flow in this tube is dictated by the needs of the gas-cooled leads between $50 \mathrm{~K}$ and room temperature. The mass flow through the shield circuit is governed by the needs of the gas-cooled leads. The short solenoid leads will need 0.05 grams per second; the long solenoid leads will need $0.035 \mathrm{~g} / \mathrm{s}$. The gas exits from the gas-cooled electrical

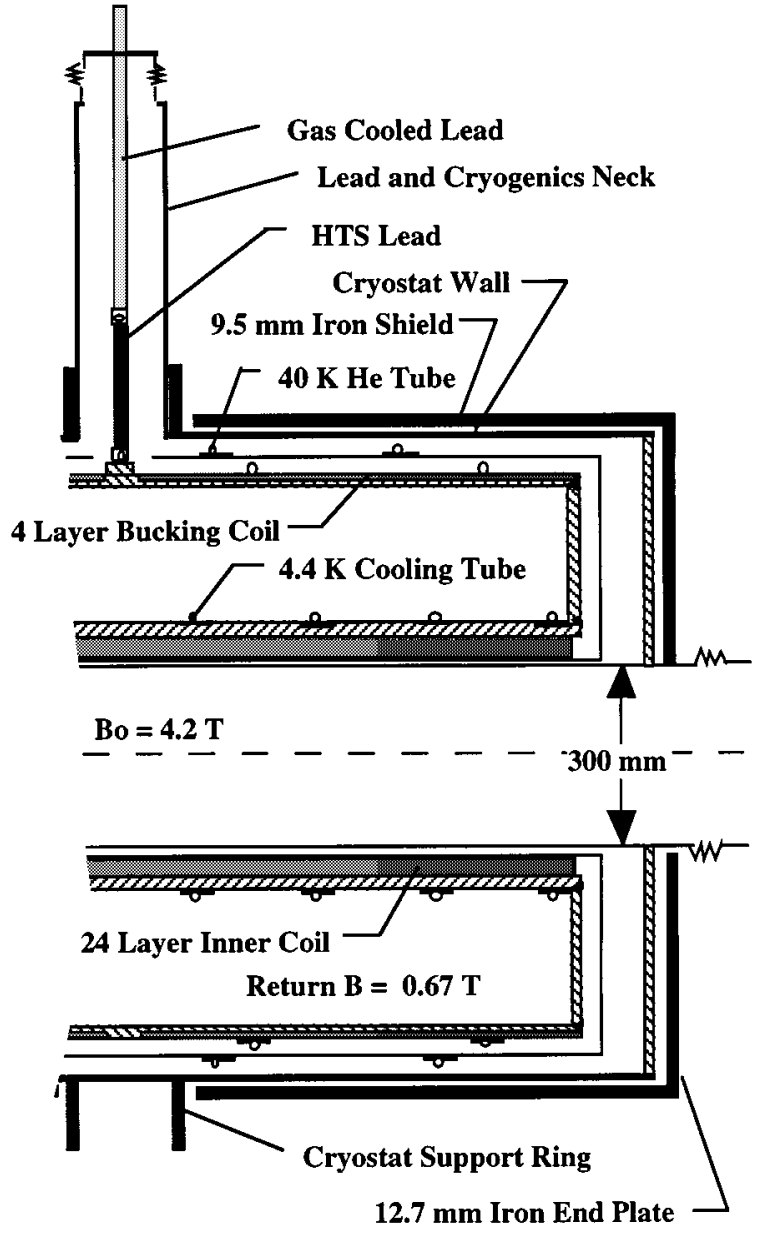

Fig. 2. A cross section of the $1.5-\mathrm{m}$ long focusing solenoid parallel to the solenoid axis.

leads at room temperature. It returns warm to the refrigerator compressor suction. In the short solenoid, the shield gas enters the gas-cooled leads at about $55 \mathrm{~K}$. In the long solenoid, the top of the HTS leads will be about $70 \mathrm{~K}$. The same HTS leads can be used for both magnets.

\section{POWER SuPPly AND Quench PROTECTION}

For simplicity sake, it is assumed that each focusing solenoid has its own power supply. A 5-V and 500-A power supply can be used for both magnets. The charge time for the short magnet, with $3 \mathrm{~V}$ across its leads is about $600 \mathrm{~s}$. The charge time for the long magnet is about $3200 \mathrm{~s}$ with $3 \mathrm{~V}$ across its leads. The power supply should allow one to charge and discharge the magnet and control its current to a set value. The magnet power supply is not required to operate the magnet at both positive and negative currents.

Quench protection for the magnet is by quench-back from the support structure and bobbin. The proposed quench protection system is completely passive. As a normal region develops in one coil, a current is induced in the adjacent coil bobbin. This current heats the superconducting coils driving them normal. Since each magnet is individually powered, a quench back quench protection system is more than adequate to protect the focusing solenoids. 


\section{COnCluding COMMENTS}

The focusing solenoids for the neutrino factory linear accelerator can be built so that stray magnetic flux does not affect the superconducting RF cavities that are about 1-m from the focusing solenoid. The superconducting solenoid can be built so that it generates nearly zero net magnetic moment at all excitations. This is the principle behind the actively shielded solenoids commonly used for MRI magnets and NMR magnets. Active shielding (zero net magnetic moment) alone will not keep stray field from the magnet from affecting the RF cavities. The distribution of the currents in the bucking coil is also important. An iron shield around the focusing solenoid will remove all of the stray flux from the magnet except for flux, which passes through the beam hole. Iron shields that are about $300 \mathrm{~mm}$ from the end of the focusing solenoid iron shield will further reduce the magnetic flux seen by the RF cavity.

The superconducting focusing solenoids can be built using commercial MRI magnet conductor. The superconducting coils will be wound and potted. They will be indirectly cooled by conduction from the support structure. The support structure will be cooled using two-phase helium carried in tubes that are attached to the support structure or bobbin.

As with modern MRI magnets, the focusing solenoids would have HTS leads between the magnet at $4.4 \mathrm{~K}$ and the shield at say $50 \mathrm{~K}$. Helium gas at $30 \mathrm{~K}$ is used to cool the magnet shield. After the shield gas cools the shield and cold mass support intercepts it can be used to cool the gas-cooled current leads that are attached to the top of the HTS current leads.

Each focusing solenoid will be separately powered. The actively shielded solenoids are protected during a quench by quench-back from the support structure and bobbin.

\section{REFERENCES}

[1] N. Holtkamp and D. Finley, Eds., A Feasibility Study of a Neutrino Source Based on a Muon Storage Ring: Fermilab-Pub-00/108E, 2000.

[2] S. Ozaki, R. B. Palmer, M. S. Zisman, and J. C. Gallardo, Eds., Feasibility Study II of a Muon-Based Neutrino Source: BNL-52 623, June 2001.

[3] M. A. Green, E. L. Black, and R. C. Gupta et al., "The role of superconductivity and cryogenics in the neutrino factory," in Advances in Cryogenic Engineering. New York: AIP, 2001, vol. 47, to be published.

[4] H. Padamsee, "Superconducting RF-New directions," in 2001 Particle Accelerator Conf. Proc., Chicago, IL, June 18-22, 2001.

[5] M. A. Green, G. F. Smoot, and R. L. Golden et al., "ASTROMAG: A superconducting particle astrophysics magnet facility for the space station," IEEE Trans. Magnetics, vol. 23, p. 1240, 1987.

[6] "A large superconducting detector magnet without an iron return path," in Supercollider. New York: Plenum, 1989, vol. 1, p. 627.

[7] F. Krienen et al., "The superconducting inflector dipole for the g-2 muon storage ring," IEEE Trans. Appl. Superconduct., vol. 5, p. 671, 1995. 